\title{
Ultra-Thin Chip Package (UTCP) and Stretchable Circuit Technologies for Wearable ECG System
}

\author{
Tom Sterken, Jan Vanfleteren, Tom Torfs, Maaike Op de Beeck, Frederick Bossuyt, Chris Van Hoof
}

\begin{abstract}
A comfortable, wearable wireless ECG monitoring system is proposed. The device is realized using the combination of two proprietary advanced technologies for electronic packaging and interconnection : the UTCP (UltraThin Chip Package) technology and the SMI (Stretchable Mould Interconnect) technology for elastic and stretchable circuits. Introduction of these technologies results in small fully functional devices, exhibiting a significant increase in user comfort compared to devices fabricated with more conventional packaging and interconnection technologies.
\end{abstract}

\section{INTRODUCTION}

$\mathbf{I}^{\mathrm{N}}$ current wearable biomedical devices comfort and unobtrusiveness are key properties for user acceptance. New packaging and assembly technologies are necessary to combine the ever increasing complexity of such devices with the desired comfort. There are two strategies to obtain unobtrusiveness in wearable devices:

- Miniaturization: the device becomes so small that it is not disturbing at all for the user and if possible not noticeable by others.

- Conformable circuitry: if the device cannot be made small, due to complexity of the device or due to its desired functionality (e.g. a large area sensing, illumination or antenna operation), then unobtrusiveness can be obtained by providing the circuit with the ability to deform in the same irregular way as the surface (skin) onto which it is applied.

In order to enhance miniaturization, an UTCP (ultra-thin chip packaging) technology is developed. To obtain conformability, original technology for stretchable and elastic electronics and sensors circuits has been developed. In this paper, the basics of both technologies are presented. As an application in which the two technologies are advantageously combined, the development of a wireless wearable ECG monitor, including design, fabrication and performance analysis, is presented too.

Manuscript received April 14, 2011. This work was supported in part by the IWT-SBO-BioFlex and the EC-FP6-SHIFT projects.

Tom Sterken, Jan Vanfleteren and Frederick Bossuyt are with the Center for Microsystems Technology (CMST), IMEC and Ghent University, Gent-Zwijnaarde, B-9052, Belgium (e-mails : Tom.Sterken@UGent.be, Jan.Vanfleteren@UGent.be, Frederick.Bossuyt@UGent.be)

Tom Torfs, Maaike Op de Beeck, and Chris Van Hoof are with IMEC, Leuven, Belgium (e-mails Tom.Torfs@imec.be, opdebeem@imec.be, chris.vanhoof@imec.be )

\section{II.UTCP TECHNOLOGY}

\section{A. Concept of Ultra-Thin-Chip-Package}

Shrinking the size of an electronic circuit on a printed circuit board (PCB) or a flexible printed circuit (FPC or flex) requires clever routing; however the miniaturization is limited by the area which is consumed by the active and passive components of the system. A further reduction of the area of the system can be reached by integrating components in the third dimension, hence by embedding either passive components [1], either active integrated circuits (ICs) in the PCB or flex. In order to do this with a successful and reliable result, the height of the embedded components should be limited to the thickness range of the glue used to laminate the PCB or flex layer, which is 25 to $50 \mu \mathrm{m}$. To fulfill this need, an Ultra-Thin Chip Packaging technology (UTCP) is developed for realizing such ultrathin embedded chips.

Except for the dimensional aspects of embedding an ASIC, the UTCP package should also bridge the gap between the high-pitched contact pads of the chip (typically less than $150 \mu \mathrm{m}$ ) and the lower resolution of the $\mathrm{PCB} / \mathrm{FPC}$ technology, in the range of $500 \mu \mathrm{m}$. This is realized by embedding the IC into an interposer of polyimide, on top of which a fan-out is fabricated using CMOS-compatible processing techniques.

The UTCP-technology presented in this work is based on the technology which was presented in [1], however the technology has been severely redesigned towards a higher compatibility with existing packaging technology. The main focus of this redesign is oriented towards a higher throughput and an improved process yield.

\section{B. Process Overview}

The UTCP process is shown schematically in Fig. 1. The first step of the UTCP technology is to reduce the chip thickness from its initial value (250-750 $\mu \mathrm{m}$, depending on the wafer fab) down to $\sim 30 \mu \mathrm{m}$, by removing the non-active bulk silicon from the backside of the IC using a grinding and polishing sequence. In parallel to this step, a release layer is deposited on top of a glass substrate used as processing carrier. On top of this release layer a polyimide layer is spin-coated and cured. The polyimide which is used (HD4110 by Hitachi-Dupont [3]) is a photopatternable type of polyimide, which is cured in a vacuum oven at $375^{\circ} \mathrm{C}$. The high curing temperatures for polyimides pose a strong limit to the material selection for 
the release layer. In this technology, a 400nm thick layer of evaporated $\mathrm{KCl}$ salt is used, which is patterned to the desired shape using a mechanical shadow mask.

Next, the thinned dies are glued to the base substrate using the thermosetting polymer BCB (Cyclotene 3022-46 from Dow [4]), which is spun on top of the substrate. Once the chip has been placed and the BCB is fully cured, a second layer of HD4110 polyimide is spun on top of the stack, and is patterned to remove the polyimide on top of the contact pads. After curing this top layer, a $6 \mu \mathrm{m}$ thick copper layer is deposited by sputtering and electroplating, and patterned using lithography and spray etching of the copper. Finally the package is released from the substrate by cutting out the shape of the final UTCP and dissolving the $\mathrm{KCl}$ release layer in water.

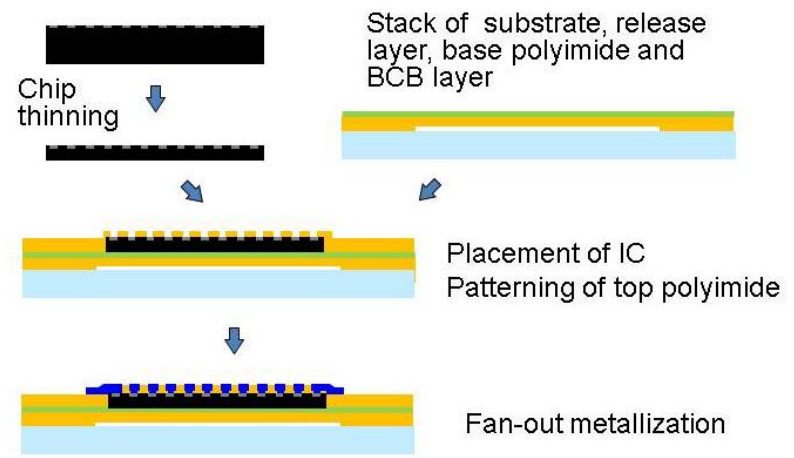

Fig. 1. Schematic overview of the UTCP technology

The use of an interposer with a fan-out introduces the possibility of testing each UTCP before embedding it in a flex, hence only known-good-dies are embedded, which is an important advantage of UTCP over most other thin chip packaging techniques. To test the UTCP's, test structures with connectors are added to the UTCP fan-out (Fig. 2). Before flex lamination, the UTCP part with test structure is removed by cutting the polyimide layers.

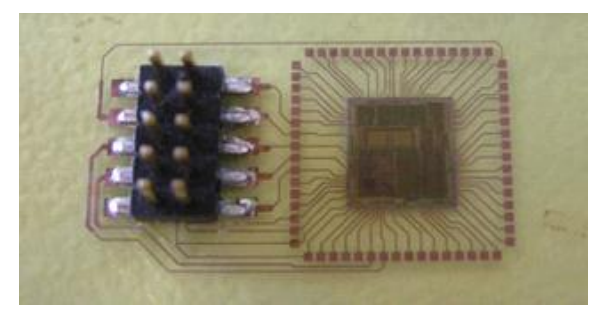

Fig. 2:

The UTCP-

packaged

MSP430F1611

has a fan-out and

a test-connector

to ensure the use

of known-good dies.

\section{StRETCHABle Circuit TeChNOLOGY}

The mainstream stretchable circuit technology was developed with potential future industrialization in mind. Also the capability for creation of high complexity circuits was pursued. These two requirements impose following consequences for the technology: use of standard packaged, off-the-shelf electronic and sensor components and use of standard Printed Circuit Board (PCB) manufacturing and assembly technologies. This requires the use of $17 \mu \mathrm{m}$ or $35 \mu \mathrm{m}$ thick $\mathrm{Cu}$ as interconnection conductor and lead-free solder assembly of the components onto the board.

Stretchability is then obtained by providing standard rigid or flexible components or component islands, and connecting these components / component islands by stretchable interconnects, as shown schematically in Fig. 3.

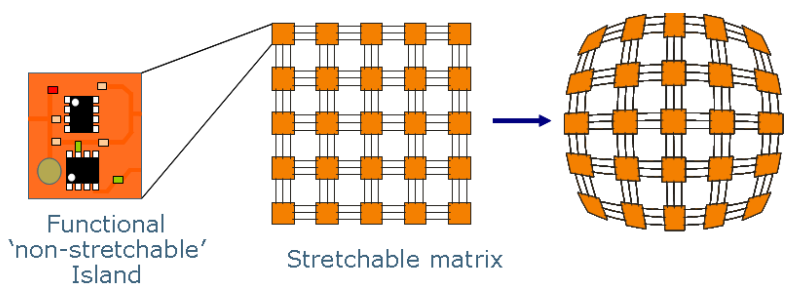

Fig. 3. Principle of stretchable circuit, composed of rigid/flexible component islands, connected with stretchable interconnections.

Such an assembly allows deforming a flat circuit, as produced in a PCB plant, into any irregular shape. This would not be possible if the circuit and the connections are only flexible and not stretchable: a flat, flexible-only circuit can be deformed to a cylinder or a cone, but not to e.g. a sphere or any irregular shape, which is possible if the circuit is stretchable. The essential challenge for developing a stretchable circuit technology is thus the creation of stretchable electrical interconnections. Stretchability and elasticity is obtained by providing the $\mathrm{Cu}$ conductors not as straight lines, as in standard rigid or flex PCB's, but as meander shaped lines instead, and secondly by embedding the whole circuit (components and conductors) in an elastic polymer material like PDMS (poly-dimethyl siloxane, better known as silicone) or PU (poly-urethane). In this way the meandered $\mathrm{Cu}$ conductor acts as a 2-dimensional spring : when stretching the meander, the spring is deformed, but the electrical connection is maintained. This proprietary developed stretchable circuit technology is called SMI ("Stretchable Mould Interconnect") and is described extensively in recent publications $[5,6]$. The technology involves standard production of an electronic circuit (lithography and wet etching of $\mathrm{Cu}$, component solder assembly) on a rigid temporary carrier, followed by a double overmolding or casting step for application of the elastic polymer, with removal of the temporary carrier between the two overmolding/casting steps. The creation of the $\mathrm{Cu}$ meanders is done either by lithography and wet etching, either by laser structuring.

Figure 4 shows a stretchable breathing monitor. The complexity of sensor makes partitioning necessary. Two smaller mechanically flexible parts are connected by a stretchable 17-conductor interconnection bus. This results in a more comfortable circuit, compared to a single large area flex circuit. 


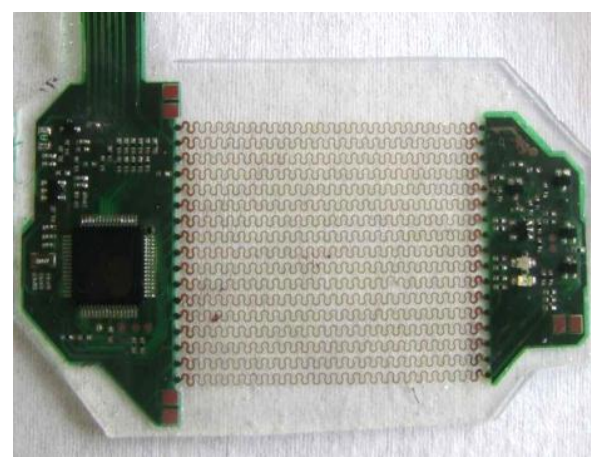

Fig. 4. Stretchable breathing monitor, consisting of 2 flexible circuit islands, interconnected with 17-conductor stretchable bus.

\section{WEARABLE ECG SYSTEM}

In previously published work a wireless ECG sensor system with an embedded UTCP-packaged microcontroller was presented [7]. The improved version presented here combines three embedded ICs with stretchable technology.

\section{A. Circuit design and lay-out}

The wearable ECG system (Figure 5) is built around three main active components:

- IMEC's proprietary ultra low power $(60 \mu \mathrm{W})$, low noise $(60 \mathrm{nV} / \sqrt{\mathrm{Hz}})$ biopotential amplifier [8] with adjustable gain and bandwidth filtering, featuring a high common mode rejection ratio $(>110 \mathrm{~dB})$ and a high differential input impedance $(>100 \mathrm{M} \Omega)$

- A commercial low power microcontroller Texas Instruments MSP430F1611 with on-chip 12-bit analogto-digital converter (ADC)

- A commercial low power $2.4 \mathrm{GHz}$ narrowband radio transceiver Nordic nRF24L01

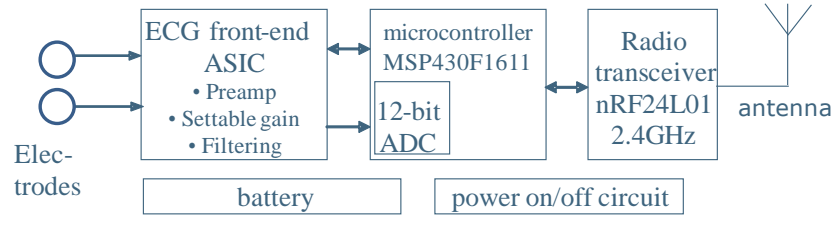

Fig. 5: Block diagram of the wearable ECG system.

The ECG signals are acquired from the body using commercial cylindrical $\mathrm{Ag} / \mathrm{AgCl}$ electrodes that protrude through the PDMS molding to make contact with the skin.

The system is powered from a compact Lithium-polymer rechargeable battery with a capacity of $20 \mathrm{mAh}$, dimensions of $18 \mathrm{~mm} \times 12 \mathrm{~mm} \times 3.0 \mathrm{~mm}$ and a mass of only $0.8 \mathrm{~g}$.

To turn the system on or off through the PDMS overmolding a compact reed switch can be activated using a magnet. In that way the need for mechanically contacting a switch or button through the PDMS is avoided while still allowing to completely power the system off for storage with only a few nA of leakage current. The contacts that allow recharging the battery protrude through the PDMS

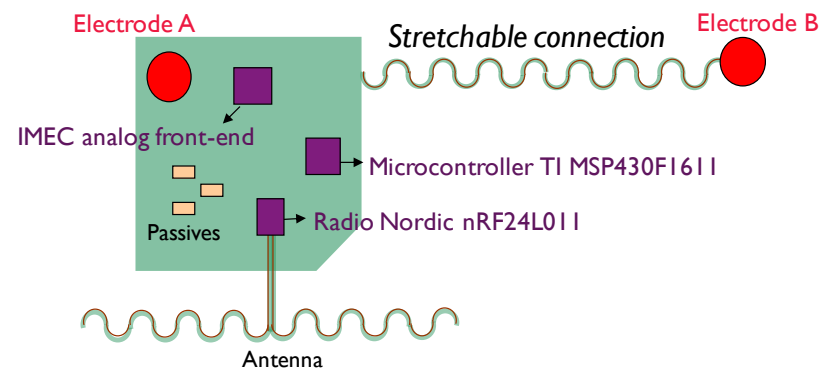

Fig. 6: Conceptual layout of the wearable ECG system (battery on bottom side not shown)

overmolding on the side away from the body, opposite of the ECG electrodes.

The conceptual layout of the building blocks in the system (Fig. 6) shows that the active components, the battery, the supporting passives and one of the ECG electrodes are integrated on one central island, implemented on a flex PCB. The second ECG electrode, which needs to be placed at some distance from the first electrode to obtain a large signal to noise ratio when the device is placed on the chest, is connected using a stretchable meander connection as described in the previous section.

The custom-designed dipole antenna has been implemented using stretchable meanders and was designed to match to the output impedance of the radio taking also into account the characteristics of the flexible circuit and the PDMS molding.

\section{B. Fabrication}

To decrease the size and thickness and to increase the flexibility of the system, the 3 ICs of the ECG-system have been packaged using the UTCP technology which was described above. The achieved yield of functional microcontrollers and radio ICs was $66 \%$ respectively $70 \%$. The analog front-end ASIC however achieved a yield of only $30 \%$. The origin of this discrepancy is still under investigation The known-good-dies are next embedded in a FPC or flex, using a commercially available process (performed by ACB [9]).

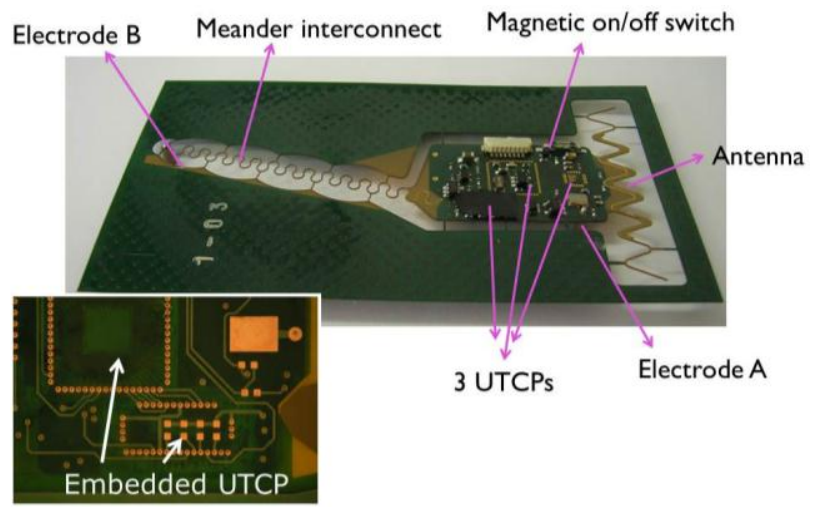

Fig. 7. The flex substrate with three embedded UTCP-packaged ICs is suspended in a frame in order coat both top and bottom sides of the circuit with PDMS, thus avoiding a seam which might turn into a reliability issue. 
In this process, the 4-layered flex is composed by laminating two 2-layered flexes using adhesive sheets. The UTCP is manually aligned on top of the patterned inner layer of one of the flexes, and next attached by locally heating up the adhesive (heat-tacking).

The process is finished by laminating the flexes, drilling and electroplating of through-holes and eventually patterning of the outer layers. A stretchable solder mask is applied. Finally, the flex is shaped using a laser: the stretchable meander and the antenna are cut out, however they are still suspended in a frame of unused flex (Fig. 7). Finally, the passives and battery are mounted on the FPC.

In order to protect the electronics, the ECG-system is covered in a PDMS jacket. At the same time, this jacket turns the meandering flex interconnect into a stretchable electrode. The result is shown in Fig. 8.

\section{Evaluation and Measurements}

The system was applied to the skin using medical-grade double-sided adhesive. To obtain good contact between the skin and the protruding $\mathrm{Ag} / \mathrm{AgCl}$ electrodes a droplet of standard electrically conductive electrode gel is dispensed on top of the electrodes prior to the placement. Research at IMEC is ongoing to allow replacement of these wet gel electrodes by dry electrodes which would greatly increase the ease of use. The stretchable meander connection between the distant electrode and the central island greatly improves the user comfort, since it conforms very easily to the body curvatures.

An example of an ECG signal as acquired by the system from the chest of a healthy adult volunteer and wirelessly transmitted to a receiving PC is shown in Fig. 8 (bottom). Although the signal-to-noise ratio of the system hasn't been measured on the embedded system, Fig. 8 illustrates the good quality of the signal.

The power consumption of the system in active mode, continuously transmitting the ECG signal, is $1.6 \mathrm{~mA}$ at $3 \mathrm{~V}$. The $20 \mathrm{mAh}$ battery will therefore allow continuous operation of the system during 12 hours before it needs to be recharged

\section{CONCLUSION}

For the wearable wireless ECG monitor demonstrated in this paper, two advanced packaging and circuit technologies have been advantageously implemented: the Ultra-thin Chip Package (UTCP) technology, including embedding in a conventional FPC (flexible printed circuit) for miniaturization and circuit thickness reduction, and secondly the SMI (Stretchable Mould Interconnect) elastic circuit technology for comfortably connecting a distant electrode and for fabrication of a stretchable antenna. The introduction of both technologies contributes to a significant increase of user comfort, comparing with rigid or 'flex-only' versions, while maintaining full functionality.
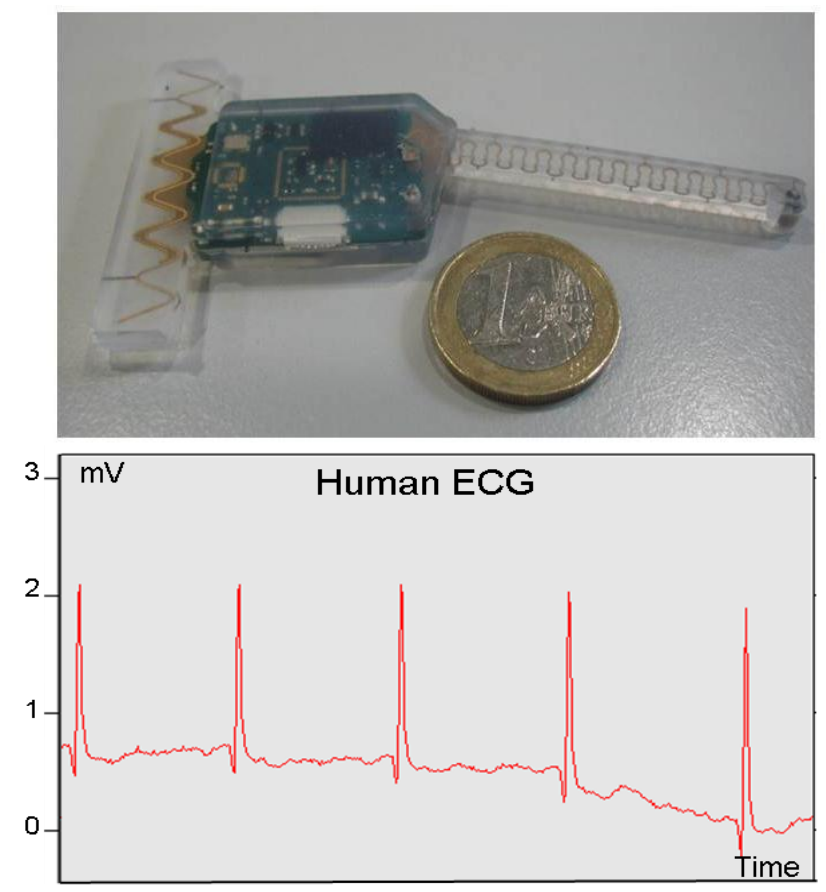

Fig. 8. Top: the finished ECG-sensor device.

Bottom: the measured ECG signal at the receiver side.

\section{ACKNOWLEDGMENT}

The prototypes presented could not have been realized without the professional assistance of An Gielen, Bart Reekmans, Liang Wang, Dieter Cuypers, Steven Brebels and Aitor Arriola.

\section{REFERENCES}

[1] Lenihan, T.; Schaper, L.; Shi, Y.; Morcan, G.; Parkerson, J.; "Embedded thin film resistors, capacitors and inductors in flexible polyimide films ", Proc. ECTC 1996, pp. 119 - 124

[2] Christiaens, W.; Bosman, E.; Vanfleteren, J.; , "UTCP: A Novel Polyimide-Based Ultra-Thin Chip Packaging Technology," Components and Packaging Technologies, IEEE Transactions on , vol.33, no.4, pp.754-760

[3] HD4100_processguide, Available: http://hdmicrosystems.com/HDMicroSystems/en_US/pdf/HD4100_ProcessGuide.pdf

[4] Cyclotene_3000_dry_etch.pdf, Available: http://www.dow.com/cyclotene/docs/cyclotene 3000 dry etch.pdf

[5] Axisa F., Bossuyt F.,Vanfleteren J., "Laser based fast prototyping methodology of producing stretchable and conformable electronic systems", Proc. 2nd IEEE ESTC Conf. (ESTC-2008), Greenwich, London, Sept. 2008.

[6] Bossuyt F., Vervust T., Axisa F., and Vanfleteren J., "A New Low Cost, Elastic and Conformable Electronics Technology for Soft and Stretchable Electronic Devices by use of a Stretchable Substrate", Proc. EMPC2009, Rimini, Italy, pp. 697-702, June 2009.

[7] T. Torfs, W. Christiaens, J. Vanfleteren, W. Huwel, W. Perdu, R.F. Yazicioglu, S. Brebels, C. Van Hoof, "Flexible wireless biopotential system with embedded ultra-thin chip", Proc. Smart System Integration Conference 2009, Brussels (Belgium), pp.536-539, March10-11, 2009

[8] R. F. Yazicioglu, P. Merken, R. Puers, C. Van Hoof, "A $60 \mu \mathrm{W}$ $60 \mathrm{nV} / \sqrt{ } \mathrm{Hz}$ readout front-end for portable biopotential acquisition systems, " IEEE J. Solid-State Circuits, vol.42, no.5, pp.1100-1110, May 2007.

[9] ACB is designing and fabricating PCB's: www.acb.be 